Cahiers de philosophie de l'université de

Levinas : au-delà du visible

\title{
De la phénoménologie du son à la phénoménologie du visage
}

Dan Arbib

\section{(2) OpenEdition}

1 Journals

Édition électronique

URL : https://journals.openedition.org/cpuc/812

DOI : $10.4000 /$ cpuc.812

ISSN : 2677-6529

Éditeur

Presses universitaires de Caen

\section{Édition imprimée}

Date de publication : 31 juillet 2012

Pagination : 101-124

ISBN : 978-2-84133-410-0

ISSN : 1282-6545

\section{Référence électronique}

Dan Arbib, «De la phénoménologie du son à la phénoménologie du visage », Cahiers de philosophie de l'université de Caen [En ligne], 49 | 2012, mis en ligne le 07 juin 2018, consulté le 01 février 2023. URL : http://journals.openedition.org/cpuc/812 ; DOI : https://doi.org/10.4000/cpuc.812

\section{(c) $(1)(9$}

Creative Commons - Attribution - Pas d'Utilisation Commerciale 4.0 International - CC BY-NC 4.0 https://creativecommons.org/licenses/by-nc/4.0/ 


\section{De la phénoménologie du son à la phénoménologie du visage ${ }^{1}$}

$\mathrm{D}$

ANS L'HISTOIRE DE LA RÉCEPTION de la philosophie de Levinas, nous bénéficions aujourd'hui d'un double avantage qui, en quelque sorte, fait époque. D’abord, un recul de la figure médiatique du philosophe qui va de pair avec la multiplication des travaux sur son œuvre: Levinas cesse d'être un nom magique, refuge facile des idéologies en mal de concepts, nom de saint invoqué dans la douleur ou la stérilité; loin d'être une faillite, ce recul est une condition favorable à la pleine éclosion des interprétations : en vertu de la loi paradoxale du rapport de proportionnalité inverse entre la présence d'un penseur et l'étude problématisée de ses textes, pareille distance, aujourd'hui effective, rend possible la problématisation et l'analyse. D'autre part, la publication d'inédits peut et doit susciter une appréciation plus subtile, plus nuancée et tout simplement plus profonde, de l'histoire des concepts et des intuitions rectrices du philosophe: il s'ensuivra à coup sûr une plus grande traçabilité des concepts, une saisie plus large du corpus, saisie diachronique, permettant de distinguer les lignes de forces, les contrastes, les pistes abandonnées ou reprises. C'est dire si, cette visibilité à peine acquise avec la publication récente des deux volumes d'inédits, tout ne fait que commencer.

Les Carnets de captivité du premier volume donnaient à voir la pensée de Levinas à l'état naissant: les contrastes s'y faisaient plus nets, les refus plus violents, les concepts plus âpres, que dans les grandes œuvres; les notes prises dans la relative solitude de la captivité faisaient voir plus dépouillées les intuitions originelles, loin de l'habillage rhétorique (même minimaliste)

1. Nous citerons toujours la conférence «Parole et Silence» à partir d'E. Levinas, Parole et Silence et autres conférences inédites au Collège philosophique (= Euvres 2), R. Calin et C. Chalier (éd.), Paris, Grasset - IMEC, 2011, p. 69-104. Nous citerons Totalité et Infini (parfois abrégé TI) dans l'édition princeps : La Haye, Martinus Nijhoff, 1961, et Hors Sujet dans l'édition du Livre de poche, 1997. 
nécessaire à la publication d'une œuvre. Apparaissait alors clairement, nous semblait-il, que toute l'œuvre postérieure, jusqu'aux concepts les plus radicaux comme la substitution, était déjà présente très tôt - de quoi donc contredire la thèse d'une évolution de Levinas ou d'une inflexion radicale de son travail; au contraire, ressortait avec force l'admirable permanence d'une intuition unique menée jusqu'à ses développements les plus aboutis. Mais ce n'est justement pas dire qu'on ne puisse, dans le développement de ces intuitions, se proposer de suivre l'histoire interne et subtile de certains motifs, et substituer au concept d'évolution le concept d'épanouissement. Les éditeurs des deux volumes nous en fournissent eux-mêmes le meilleur exemple. Dans la préface du second volume, Rodolphe Calin et Catherine Chalier ont par exemple parfaitement suivi la manière dont la genèse de Totalité et Infini se donne à lire comme une explication avec la Geworfenheit heideggérienne, comment le mouvement vers l'au-delà se conceptualisait initialement comme métaphore, avant l'abandon de ce concept.

C'est à un exercice de ce type qu'on voudra ici s'employer, à partir de la considération d'un fait: les deux premiers volumes proposent - et le second de façon thématique - de subtiles analyses sur le son. Ces analyses, surprenantes par ce qu'elles anticipent, semblent toutefois demeurer sans postérité dans l'œuvre ultérieure. Or un tel paradoxe est plutôt l'énoncé d'une question: comment la phénoménologie du son ouvre-t-elle sur les analyses à venir? Il s'agira donc d'étudier la structure et la postérité de l'analyse du son, entamée p. 89 des Euvres 2 dans la conférence intitulée «Parole et Silence» donnée en 1948 au Collège de philosophie. Quelque tentante que soit l'ambition de poursuivre l'analyse jusqu'à Autrement qu'être ou au-delà de l'essence, nous nous donnerons pour terminus ad quem la date de 1961, puisque les inédits publiés s'arrêtent à la publication de Totalité et Infini. Notre hypothèse, annonçons-la d'emblée: le son anticipe autant qu'il endosse déjà en 1948 la fonction qui reviendra au visage dans Totalité et Infini; par là la phénoménologie du son serait une annonce, un premier «coup d'essai» d'une phénoménologie de l'extériorité que le visage fera définitivement aboutir.

\section{Le son dans «Parole et Silence» (1948)}

Suivons la conférence de 1948. Tel qu'il y apparaît, il fait l'objet d'une quadruple étude: 1 / le son est d'abord décrit comme fondamentalement dual, avant d'être 2/ analysé comme événement. Ces deux premières déterminations permettent de faire du son $3 /$ « la gloire de l'événement autre », ouvrant sur $4 /$ une genèse du langage. Reprenons ces quatre moments pas à pas. 
1/ Levinas explore d'abord l'aspect dual du son ${ }^{2}$ : d'un coté, le son est une sensation, et à ce titre appartient au monde de la lumière et se réfère au sujet; d'un autre côté, il est «rupture dans le monde de la lumière». Ces deux aspects ne font pas l'objet de la même attention, puisque le son comme rupture fait l'objet d'une élaboration accrue. (a) D'une part, comme sensation, le son fait partie du «monde de la lumière». Pourquoi? Parce que comme la lumière, le son procède du monde ouvert par le sujet, c'està-dire de la phénoménalité du monde; à ce titre le son et la vision sont des sens analogues: «Il [le son] se réfère, comme toute lumière - au sujet. Venant du dehors, entendu, il est comme s'il venait de nous» (p. 90). Le son, entendu comme s'il venait de nous, est alors décrit (il faut le noter) comme l'objet éclairé par la lumière dans Le Temps et l'Autre: «L'objet éclairé $[. .$.$] , on le rencontre comme s'il sortait de nous { }^{3}$. Ainsi, entre le son, la vision et la compréhension, s'instaure l'identité de l'immanence, qu'indique la référence à l'évidence cartésienne: le monde de la sensation est un monde de représentations claires et distinctes: claires et distinctes parce qu'elles procèdent de moi, parce qu'elles me font face comme si je les projetais moi-même. La sensation n'ouvre ainsi aucune transcendance: monde clos donc de la possession, de la maîtrise, de la compréhension ${ }^{4}$. Parce qu'il est sensation, c'est-à-dire paradoxalement vision, le son demeure dans la sphère proprement immanente de la phénoménalité mondaine. Mais (b) la sonorité du son ouvre sur un en-dehors de la sensation, c'est-à-dire un en-dehors de l'immanence. La "sonorité du son», c'est ce qui, dans le son, fait son - par opposition au son comme sensation. Le son comme sensation était son à partir de moi, puisqu'il était reconduit au sujet de la lumière ouvrant le monde - bref, pourrait-on dire, par un raccourci que nous autorise Levinas, au Dasein dévoilant. Mais le son décrit comme son, c'est-à-dire dans sa sonorité de son, advient à partir de lui-même - c'est-àdire finalement hors du monde phénoménal. Le paradoxe est ici que c'est en obéissant à la plus stricte définition heideggérienne du phénomène comme ce qui apparaît lui-même à partir de lui-même $\left(\$ 7\right.$ de Sein und Zeit $\left.{ }^{5}\right)$ que le

2. Cette structure est sans doute inspirée à Levinas par des Leçons pour une phénoménologie de la conscience intime du temps de Husserl; mais nous ne pouvons ici qu'indiquer un rapprochement qui reste à construire.

3. Le Temps et l'Autre [1946-1947], Paris, PUF (Quadrige), 1983, p. 47.

4. Voir entre autres: «Le monde de la lumière est un monde de transparence à travers lequel nous possédons le monde en l'embrassant » (p. 90); « tout ce qui est autre est à la fois mien » (ibid.).

5. La différence entre Heidegger et Levinas demeure pourtant: que Heidegger pense le phénomène comme visible (Voir Être et Temps, $\$ 7 \mathrm{~A}$, et la dérivation du phénomène à partir de la lumière, phôs) et donc maintient, et même explore sa détermination grecque - détermination dont toute l'œuvre de Levinas fera la critique. 
son ne relève pas de la phénoménalité mondaine et récuse la catégorie très heideggérienne de dévoilement. Qu'est-ce que le son en lui-même? Non plus ce qui (s')inscrit dans la phénoménalité de l'immanence toujours mienne, mais ce qui provient de l'au-delà du phénomène, ce qui rompt l'achèvement du monde - bref, «rupture», «éclat», «scandale». Trois concepts qui disent la trouée opérée dans le monde par le son: (i) car certes l'éclat est lumière et il s'agit de percer la lumière pour sortir d'elle; mais l'éclat est ce qui, lumière extrême, offusque la lumière même, visibilité aveuglante trouant la visibilité, trou noir hors de toute apparition, point aveugle; (ii) scandale, métaphore «sociale» d'après Levinas, puisque le son, en rompant l'ordre de la phénoménalité, brise le monde du dévoilement et le perce, anarchie dans la phénoménalité (anarchie dont on devine qu'elle sera bientôt celle du Dire) : «rupture». (iii) Mais attention: il ne s'agit pas d'une rupture au sein du monde de la lumière, comme si ce monde était lui-même fracturé (une telle rupture n'en serait pas une, puisque, dernière ruse de l'immanence, elle ne briserait l'unité du Même qu'en apparence, en recollant toujours les bris sous l'unité de la lumière), mais d'une rupture advenant de l'hors du monde, trouée, extase, déchirement: «le son déchire le monde de la lumière» (p. 9o). Ainsi le son est-il structurellement double: il est du monde, mais advient du dehors: à la fois dedans et dehors. Dedans en tant que qualité sensible, phénomène, lumière; mais, dehors, parce que, en lui, «le monde éclate", se déchire et s'ouvre. Le son ne fait pas signe vers l'autre du monde mais fait éclater le monde en son sein même: il contient donc plus qu'il ne le peut, puisqu'il contient l'hors du monde depuis le monde lui-même. Ce qu'annonce le son n'est pas l'autre visible (l'autre monde), mais l'autre $d u$ visible (l'autre du monde) - la gloire d'un être autre.

2/ À cette première caractéristique du son (sa structure duale), s'en joint une seconde: le son comme événement. Pour comprendre cette seconde caractéristique, il convient de partir d'un trait tout particulier: (a) La superfluité du son. En effet, le son diffère de la couleur et de la forme, de la saveur, de l'odeur, du contact, en ce qu'il est une qualité superflue: non pas parce que les autres qualités secondes seraient «adhérentes» (la couleur l'est, à la rigueur, mais l'odeur?), mais parce que le son dit le dérangement de la chose. Le son vient donc en surcroît aux «manifestations de la chose», c'est-à-dire à la sensorialité générale. Le bruit double l'action et à ce titre le son est épiphénomène: annonce, porte-voix, des manifestations de l'être. Ainsi (b) le son est-il inséparable de la verbalité de l'être, de la dimension d'action des étants: «Résonner, c'est nous imposer ce qu'il y a de verbe dans tous les substantifs» (p. 91): le son ne colle pas à la chose, il en dit l'événementialité. C'est pourquoi (c) le son est le temps: «le son roule le temps lui-même» (ibid.), et non pas sur le temps: en effet, le son est temps, 
temps phénoménalisé, alors que la couleur, par exemple, est à travers le temps, sans être ce temps qui roule sur elle. S'autorisant d'un poème de Pouchkine, Levinas fait du temps l'événement même de l'être donné par le son. On ne manquera pas de remarquer ici le bergsonisme sous-tendu par une telle description, Levinas n'ayant par ailleurs jamais manqué de reconnaître à Bergson le mérite d'avoir fait entrevoir le dynamisme de l'être.

3/ Si le son est à la fois rupture de la phénoménalité à même le phénomène et pure événementialité, alors la conjonction des deux déterminations (le son comme rupture, le son comme événement) aboutit à la corrélation: «Le son est donc la gloire de l'événement autre» (p. 92). Il faut bien comprendre que c'est là une conclusion de deux déterminations initiales, étudiées chacune séparément. Mais on comprend que le son est alors verbe de l'autre - verbe qui s'oppose à une détermination du son comme signe et comme image. Le son n'est pas signe, car la signification s'établit toujours au sein du monde, entre des étants intramondains (Heidegger l'a bien montré au $\$ 17$ de Sein und Zeit ${ }^{6}$ ), alors que le son renvoie à l'extérieur du monde, à l'au-delà du monde, hors tout dévoilement; mais le son n'est pas non plus image de l'événement autre, puisqu'il en est plutôt le retentissement: le son n'imite pas l'autre du monde, il le bruisse. Ni signe, ni image, le son est plutôt symbole, non pas symbole au sens de la linguistique (le symbole comme signe), mais comme rapport à l'exister-autre, donc comme rapport à la verbalité de l'altérité advenue dans son événement.

4/ Si le mot est d'abord un son, il doit être possible de repérer le moment où le son vire en mot - ce que Levinas appelle «déduire la modification essentielle du son en mot» (p. 92). Si le mot est d'abord son, si le son est le retentissement de l'être, alors le moment de ce virage fera voir avec exactitude "la place exceptionnelle du langage dans l'économie de l'être». Levinas décrit la pente immédiate à laquelle cède tout son: tout son «est aussitôt qualifié», dit-il (p. 92). Qu'est-ce que cette qualification ? C'est l'intégration dans un ensemble, constitution d'une musique ou d'un rythme, par lequel le son nous devient familier et son essence de rupture est niée. Qualifier le son revient à se l'adapter, à se l'acquérir, le lier au monde des choses et des objets qui sont nôtres et que nous manipulons; dès lors si, dans les bruits du monde, la qualité l'emporte sur la sonorité, la continuité l'emporte sur la rupture. La qualification contredit la sonorité du son, parce

6. Voir Être et Temps, $\$ 17$, trad. E. Martineau, p. 82: «Le signe est un étant ontiquement à-portée-de-la-main qui, en tant que cet outil déterminé, fonctionne en même temps comme quelque chose qui indique la structure ontologique de l'être-à-portée-de-la-main, de la totalité de renvois et de la mondanéité». D'où la différence entre renvoi et signe, le renvoi étant «au fondement du signe» (ibid., p. 83). 
qu'elle est une forme de rapatriement du son sur le visible, donc sur la sphère du Même. Ainsi le son se trouve-t-il écartelé entre sa qualité (qui le rabat sur l'immanence) et sa sonorité (qui troue cette immanence). Le couple qualité / sonorité rejoue la structure duale du son en indiquant la tendance commune de la sonorité à se perdre en qualité. Certes, un éclat particulier peut encore, dans certains cas, reconquérir la sonorité contre la qualité: le son de la cloche qui tinte m'advient de l'outre-monde, et se refuse à la qualification 7 . Le son esthétisé, qualifié, c'est-à-dire mondanéisé, peut toujours re-devenir son, c'est-à-dire reconquérir sa sonorité sur sa qualité.

On pourrait croire que le mot est rattaché au son qualifié, musical, au son «intégré», puisqu'en apparence le langage tisse les réseaux qui servent à enserrer et à constituer la mondanéité du monde. Or justement, il n'en est rien: le sens du mot réside dans le fait qu' « un objet peut nous venir du dehors». Le langage ouvre sur l'extériorité à partir de la sonorité du son: «Le langage, c'est la possibilité pour un être d'apparaître du dehors». Ainsi le langage entendu comme enseignement par autrui n'est-il possible que par la dimension de sonorité du son, c'est-à-dire sa fonction de rupture. Le langage comme enseignement repose sur l'essence du son comme sonorité, puisque toute genèse du langage impose d'en revenir au son comme rupture de la phénoménalité au sein de la phénoménalité, c'est-à-dire ouverture à la transcendance.

\section{Le son hors-jeu (1948-1961)}

Phénomène hors phénomène, événement de l'altérité, verbe - le son est ainsi à l'articulation entre l'immanence et la transcendance: il est un Janus, dont une philosophie de la transcendance a besoin dans son mouvement vers l'au-delà. Pourtant, autant les développements sur l'enseignement seront importants dans l'œuvre ultérieur, autant la postérité des analyses sur le son est discrète. Cette postérité équivaut-elle à une absence? Rien n'est moins sûr, s'il est vrai qu'au son il revient de transpercer la phénoménalité pour l'au-delà du monde. Un détour permet de le comprendre. Il est un point que les Carnets de captivité ne permettent plus de remettre en doute: que l'entreprise de Levinas consiste à substituer le Juif au Dasein : «Partir du

7. On peut toutefois hésiter devant la pertinence descriptive d'une remarque comme: «La cloche est un instrument à produire du son dans les fonctions du son» (p. 93): car une cloche peut par exemple travailler à rendre le monde familier en définissant le temps et l'espace de l'action humaine, et c'est bien ce à quoi servent la plupart de celles des clochers de France: non pas briser la sphère du monde, mais l'établir et s'y inscrire. À moins que Levinas ne décrive ici le son du shofar, qui a effectivement pour but dans la tradition juive de provenir d'un antique au-delà et d'appeler l'individu à un «réveil» intérieur? 
Dasein ou partir du J.» ${ }^{8}$. Avant même que, dans la grande explication de Levinas avec Heidegger, le concept de substitution ne réponde au concept de sollicitude 9 , l'opposition apparaissait nette, précoce et immédiate, énoncée en termes Dasein / juif. Cette opposition revenait à promouvoir la révélation comme concept ultime, contre le dévoilement heideggérien, et donc à en finir avec la lumière de l'ouvert, assimilé à l'ouverture de l'Être / Néant de Heidegger réinterprété comme il y $a^{10}$. Aussi bien, Le Temps et l'Autre montrait déjà que la vision n'ouvrait à proprement parler sur aucune transcendance. Ainsi, la nécessité d'échapper à l'immanence ne peut passer que par l'ouverture, la saillie, hors de la sphère du monde phénoménal - c'est-à-dire, et c'est là le point capital, hors de la visibilité. Or seul le son en sa sonorité ouvre cette saillie et rompt «de haut» l'être de l'étant, rupture qui n'a plus rien d'une divisio entis médiévale, puisque, même en divisant l'étant entre fini et l'infini de la plus grande distance possible, la divisio entis de Scot ou Suarez continuait de promouvoir une division de l'étant, au sein de l'étant, tel que fini et infini étaient avec l'étant des transcendantaux toujours convertibles ${ }^{11}$. En 1948, seul le son ouvre sur une rupture plus radicale, séparation non de l'étant, mais avec l'étant, quoique toujours depuis l'étant. Dans ces conditions, le son pouvait-il disparaître, ne laisser aucune postérité dans l'œuvre? Non, sans doute, puisqu'une telle disparition eût signé la fin et l'impossibilité de la transcendance. Mais une telle réponse $a$ priori impose de se confronter à la disparition ou à la raréfaction au moins lexicale du mot «son» et de ses dérivés grammaticaux, et discerner, sous les changements de mots, la permanence du concept.

Indiquons d'emblée une fausse piste évidente: le son, ni bruit ni silence, ne devra pas se chercher du coté de l'il $y a$, bien que celui-ci fasse du bruit, le fameux «remue-ménage». Si Levinas insiste beaucoup sur le bruit de l'il $y a$, jamais, à notre connaissance, il n'évoque un son émané de l'il $y a$. Et on comprend pourquoi: le bruit de l'il y a n'appelle à aucune échappée, il est la

8. Carnets de captivité, in E. Levinas, Carnets de captivité, suivi de Écrits sur la captivité et Notes philosophiques diverses (= Euvres 1), R. Calin et C. Chalier (éd.), Paris, Grasset - IMEC, 2009, p. 75 . Il nous semble que «J.» abrège «Juif» et non «judaïsme», contrairement à ce que proposent les éditeurs.

9. Voir J.-L. Marion, «La substitution et la sollicitude. Comment Levinas reprit Heidegger», in Emmanuel Levinas et les territoires de la pensée, D. Cohen-Levinas et B. Clément (dir.), Paris, PUF (Épiméthée), 2007, p. 51-72.

10. "Au dévoilement de l'être en général, comme base de la connaissance et comme sens de l'être, préexiste la relation avec l'étant qui s'exprime; au plan de l'ontologie, le plan éthique» (Totalité et Infini, p. 175).

11. La divisio entis fini / infini s'insère dans les Disputationes metaphysicae de Suarez exactement à la frontière entre l'ontologie générale et les ontologies régionales de la metaphysica specialis (Disputatio XXVIII). 
sourdine mate du coquillage à notre oreille, l'immanence pure; dès lors, il se confond avec le silence lui-même, absence de toute parole, silence de la nuit, silence d'une vision muette; et le son ne peut pas être ce silence. Cette fausse piste écartée, si on restreint l'enquête - ce qui est mon projet - aux textes allant jusqu'à 1961, la postérité des analyses de 1949 sont maigres: elles se limitent à une répétition et à une contestation au moins apparente.

Soit la répétition, illustrée par un texte tout à fait contemporain de «Parole et Silence», puisque paru en 1949 dans Les Temps Modernes, repris en 1981 dans le numéro 3-4 de L'Ire des vents ( A Autour de Michel Leiris »), puis dans Hors Sujet en 1987: "La transcendance des mots. À propos des Biffures ». Ce texte se donnait jusqu'à présent comme un hapax dans le corpus lévinassien, et ce n'est pas le moindre mérite des inédits que de lui avoir rendu, si l'on peut dire, son homologue oral dans la conférence de 1948. Tout invite, nous semble-t-il, à faire du texte de la conférence "Parole et Silence» un texte préparatoire ou source de l'article sur Michel Leiris. Levinas y fait du mot le Janus déjà élaboré dans «Parole et Silence» et y construit de manière extrêmement rigoureuse l'opposition entre la vision et le son, le synchronique et le diachronique - opposition qui s'analyse comme opposition entre le mot-biffure et le mot-son. 1/ Biffure: Levinas propose d'y voir l'essence de la pensée: "la pensée est essentiellement biffure - c'est-à-dire symbole» (p. 198-199). Comment comprendre ce symbolisme de la pensée révélée par la biffure? On peut élucider plusieurs caractères de la biffure. (a) Symbolisme par inhérence. Que veut dire le fait que la pensée soit symbolique? «Les idées peuvent s'accrocher les unes aux autres et former un réseau d'associations » : chaque mot fait réseau, contient d'autres mots spontanément suggérés ou produits par les circonstances, les ressemblances sonores, la forme écrite du mot; mais «ce réseau vaut, non point parce qu'il fait passer d'une idée à d'autres, mais parce qu'il assure la présence d'une idée dans l'autre» (p. 199). Ainsi la biffure comme principe de la pensée permet de saisir la pensée «pardelà les catégories classiques de la représentation et de l'identité» (p. 199). Est-ce à dire qu'il faut ici voir dans chaque mot un débordement vers d'autres mots, à la manière dont Bergson thématise le débordement de la pensée par elle-même? Non, car (b) la biffure est déploiement du synchronique, alors que la pensée bergsonienne est pensée du diachronique ${ }^{12}$. La biffure n'est donc pas le lieu d'un déploiement temporel de la pensée et de ses associations d'idées, mais au contraire de la co-présence, en un même temps, des mots et des signifiants. C'est pourquoi elle n'est pas sortie hors de l'être présentement

12. Voir «la conception de Bergson consiste à se représenter sous forme de devenir cette négation de l'identité. L'originalité de la notion de biffure revient à poser le multiple comme simultané» (p. 199). 
présent et du principe d'identité: loin d'esquisser une quelconque sortie de l'ontologie par une promotion du futur, le symbolisme de l'inhérence annule son dynamisme propre en s'empêtrant dans la contemporanéité. La biffure est le lieu paradoxal du symbolisme ontologique, où les idées se superposent, s'entre-pénètrent, mais dans la permanence du présent, et non, comme chez Bergson, dans le mouvement continu de création de nouveauté. Ainsi, le réseau de la biffure ne s'extasie pas hors du présent, mais se déploie à même le présent. Co-présence synchronique, règne du présent, les biffures (3) sont donc installation dans l'espace et non rythme temporel. La biffure est donc prévalence de l'espace sur le temps. Dès lors (4) «ce que le jeu de biffures comporte de spatial ne tient-il pas à ce qu'il comporte de visuel?» (p. 200), se demande Levinas. En effet, la biffure n'est spatiale que parce qu'elle est d'abord une expérience visuelle, expérience visuelle «à laquelle la civilisation occidentale réduit en fin de compte toute vie spirituelle». Ainsi la biffure «a affaire aux idées, elle est lumière, elle recherche la clarté et l'évidence. Elle aboutit au dévoilé, au phénomène. Tout lui est immanent» (p. 200). La biffure est donc au lieu du Même, lieu du monde ${ }^{13}$, parce qu'elle est déploiement de l'inhérence synchronique, spatiale et visuelle ${ }^{14}$. Mais, nous l'avons vu, le son est fondamentalement autre chose et peut reconquérir sa sonorité propre contre son être de biffure: il est «rupture du monde toujours achevé de la vision et de l'art», «retentissement, éclat, scandale», trois termes repris à «Parole et Silence»; "Alors que, dans la vision, une forme épouse le contenu et l'apaise, le son est comme le débordement de la qualité sensible par elle-même, l'incapacité où se trouve la forme de tenir son contenu - une véritable déchirure dans le monde» (p. 201). Les considérations de «Parole et Silence» sont ici réintégrées dans une méditation sur le son comme fond du mot, déployant à la fois sa nature duale, sa temporalité, son événementialité et son altérité. On comprend dès

13. «Toute vision au-delà du donné demeure dans le donné. La vision est relation avec l'être, telle que l'être atteint par elle apparaît précisément comme monde» (ibid.).

14. Il faut insister sur la vision, à propos de laquelle ce texte nous permet trois précisions définitives: (a) la vision et le monde sont corrélatifs : la vision est la condition de possibilité du monde («toute vision au-delà du donné demeure dans le donné», p. 199) et en retour est vu ce qui est $d u$ monde; (b) la vision et l'art sont corrélatifs : la primauté du visuel fonde l'universalité de l'art, qui se distingue toujours par son silence («Sur ce primat de la vision repose aussi l'universalité de l'art. Il fait la beauté dans la nature, il la calme, il l'apaise», p. 201); (c) Cette co-appartenance du visuel, du monde et de l'art, permet d'affirmer que toutes les sensations sont du visuel pour autant qu'elles sont du monde. D'où le paradoxe du son: en tant qu'il est du monde, il est du visuel, et donc, en tant qu'il est art, il est silence. Levinas parvient à penser le son comme du visuel et du silence, comme art. Ces trois déterminations entérinent la détermination de la lumière comme élément occidental de la vision: la biffure est de l'ordre de l'idée claire et distincte cartésienne, du dévoilement heideggérien, de l'il y a pétrifié dans la statue. 
lors que si, d'un côté, le son comme lumière se donnait comme élément de l'art, de l'il y $a$, du silence neutre de l'être impersonnel, c'est-à-dire comme élément de l'Occident dévoilé par le comprendre du Dasein, d'un autre coté, le son comme déchirure du monde récuse l'art et le silence des espaces infinis, suppose révélation et non dévoilement et part, non plus du Dasein, mais de l'étant sans nom que les Carnets de captivité avaient pourtant déjà nommé Juif.

Qu'en est-il du son dans Totalité et Infini? L'index Ciocan-Hansel donne seulement deux occurrences du son, deux occurrences du verbe sonner dont l'une (p. 9) est introuvable et l'autre insignifiante, et aucune occurrence de sonnerie ou sonore. C'est donc peu de dire que le son ne sature pas l'ouvrage: de 1948-1949, où le son apparaissait comme le marqueur de transcendance au sein de la phénoménalité, au magnum opus de 1961 qui semble tout simplement oblitérer le son au moment d'évoquer la transcendance, la pensée lévinassienne semble s'être définitivement détournée du son; davantage: l'analyse du son de 1948 s'y trouve tout simplement contredite. Suivons donc les deux occurrences du son, p. 99 et p. 161: (a) Totalité et Infini, p. 99: la première occurrence apparaît dans la seconde section de l'ouvrage, «Intériorité et économie», et plus précisément dans les pages consacrées à la représentation («Jouissance et représentation»). Levinas y développe «la structure de la représentation comme détermination nonréciproque de l'Autre par le Même» (p. 98-99). La représentation s'oppose à tout mouvement de transcendance, puisque «dans la représentation, le moi perd précisément son opposition à un objet», opposition qui «s'efface pour faire ressortir l'identité du moi malgré la multiplicité de ses objets». La représentation ressortit donc au Même, et déploie son identité à soi: «Rester le même, c'est se représenter» (p. 99). Or c'est ici qu'intervient le son: «Le sujet qui pense par la représentation est un sujet qui écoute sa pensée : la pensée se pense dans un élément analogue au son et non pas à la lumière» (p. 99). Le son est donc ce qui du sujet lui advient à lui-même, sa musique intérieure en quelque sorte. Dès lors, il se laisse triplement déterminer: (i) il est la voix de l'universel en moi: «Le moi particulier se confond avec le Même, coïncide avec le "démon" qui lui parle dans la pensée et qui est la pensée universelle» (ibid.). Rien donc d'une transcendance: la représentation est donc le lieu où le moi s'adjoint à la totalité, à l'universel; (ii) le son est recueilli dans une écoute, non pas écoute de l'autre, mais écoute de soi, de sa spontanéité paradoxale car toujours surprenante, que Levinas appelle «génialité» ${ }^{15}$. Du coup, en dépit de cette surprise, ou plutôt

15. «Sa propre spontanéité est comme une surprise pour le sujet, comme si le moi surprenait ce qui se faisait en dépit de sa pleine maîtrise de moi» (p. 99). 
parce qu'elle est surprise de soi en soi, (iii) le son n'est pas rupture mais continuité, par quoi les moments du temps, loin d'offrir à un sujet extatique une sortie hors du présent, se fondent en une éternité pétrifiée: « retour dans la pensée présente au passé de la pensée, assomption de ce passé dans le présent; dépassement de ce passé et de ce présent, comme dans le souvenir platonicien où le sujet se hisse à l'éternel» (p. 99). Les figures de Platon et de Socrate (par l'allusion au "démon») habitent ces pages où la génialité du sujet est génialité de l'universel: «le moi qui constitue se dissout dans l'œuvre qu'il comprend et entre dans l'éternel» (p. 99). La mutation par rapport à la conceptualité de 1948 est ainsi indiscutable: en 1948, le son était doublement déterminé comme rupture du Même et advenue de l'événement autre; en 1961, il s'agit non plus littéralement d'advenue, mais de surprise, et surtout d'une fausse surprise puisqu'elle est surprise du soi par soi, du je par l'universel: le son n'est plus le son venu d'ailleurs, il est le son de sa propre pensée hissée «à l'éternel» (p. 99), le son de la pensée universelle; elle s'associe ainsi la maïeutique (comme l'indique l'allusion à Platon), par opposition à l'enseignement par/ de la transcendance prise en charge par l'innéité de l'idée cartésienne d'infini. De 1948 à 1961, l'analyse du son ne s'est pas seulement affaiblie, elle a été contredite. Le son n'est plus sortie vers l'infini, mais voix de la totalité. (b) Soit à présent la seconde occurrence, p. 161: nous sommes au tout début de la troisième partie («Visage et extériorité»), dans la section intitulée "Visage et sensibilité». Levinas y revient sur l'acquis de la seconde partie consacrée à la jouissance. La jouissance permet de contester le «caractère construit» de la sensation, «l'analyse classique » reprise par «l'idée de l'intentionnalité»: selon cette analyse, «nous nous trouverions toujours auprès des choses, la couleur est toujours étendue et objective, couleur d'une robe, d'un gazon, d'un mur - le son, bruit de la voiture qui passe, ou voix de l'homme qui parle» (p. 161). Or, ajoute Levinas, «cette critique de la sensation méconnaît le plan où la vie sensible se joue comme jouissance. La sensibilité n'est pas une objectivation qui se cherche» (p. 161). Il est possible de penser une "sensation pure», "même dans le domaine de la vision ou de l'audition», propose Levinas. C'est ici que l'analyse s'infléchit et semble retrouver certains traits de la description de 1948: proposer une «sensation pure», c'est envisager «la spécificité de chaque sensation réduite précisément à cette "qualité sans support" que les sensualistes y cherchaient, et [qui] indique une structure qui ne se réduit pas nécessairement au schéma d'un objet doué de qualités». Or, on s'en souvient, Levinas décrivait dans «Parole et Silence» un son sans support, puisque qualité toujours surérogatoire de l'objet, non attaché à l'objet. Que déduire de ce rapprochement? D'une part, ce qui était la prérogative du son seul est en 1961 la prérogative de toute la sensorialité; mais d'autre part 
surtout, ce rapprochement donne à voir l'inflexion essentielle, par laquelle l'analyse de 1948 se trouve en son fond contredite: cette sensorialité non objectivante indique, dans Totalité et Infini, non pas la transcendance, mais la jouissance de l'élément, puisque l'élément est bien «une qualité sans substance» (p. 108). Autrement dit, alors que le son permettait en 1948 de sortir de la phénoménalité du monde par la transcendance, il est en 1961 rabattu sur la sensibilité, sur la jouissance et partant sur l'intériorité non ouverte sur la transcendance de l'Autre: marqueur de transcendance horssensibilité en 1948, il est moment de la sensibilité et donc d'immanence en 1961. Là encore, l'analyse de 1961 contredit littéralement l'analyse de 1948.

Un point est donc acquis: avec l'ouvrage de 1961, le son a définitivement perdu sa fonction de transcendance, du fait de son interprétation comme moment de la sensibilité, laquelle est annexée à la sphère du moi. Ce qui revient à dire que désormais il faudra sortir de la sensorialité pour avoir accès à la transcendance, et du coup ne plus en passer par le son.

\section{La relève poétique}

Pourtant cette conclusion - la disparition du son comme marqueur de transcendance - exige l'examen par un autre biais. Rappelons que, dans «Parole et Silence», il revenait au son de possibiliser le langage; mais dès lors, l'avenir du son ne peut-il pas se trouver du côté de l'essence même de la langue? Dans cette hypothèse, la thèse de la transcendance du son dans le mot trouverait son aboutissement dans l'analyse du langage: si le son vire en mot, c'est-à-dire en langage, si le son possibilise le langage en «virant au mot», alors il se pourrait en effet que l'avenir du son soit dans l'essence du langage. Or où chercher l'essence du langage? Dans la poésie. Trois figures permettent de valider cette hypothèse: (a) Claudel, (b) Rimbaud, (c) Celan. (a) Dans un article de 1969 sur Claudel, paru dans le Bulletin de la Société Paul Claudel (janvier-mars 1969) et repris dans Difficile liberté ${ }^{16}$, Levinas, examinant l'évolution du sentiment du poète à l'égard des Juifs, soutenait que l'Holocauste faisait des Juifs les martyrs et que «tout survivant [...] est obligé à l'égard d'Israël pour des raisons qui obligent tout homme» (p. 203). Cette obligation pouvant contredire les exigences de la politique, Levinas concluait: "Mais la politique constitue-t-elle la trame ultime de l'être et le guide unique de l'action? La vision poétique qui la transcende est-elle à jamais vouée à demeurer "belles lettres" et à perpétuer les fantasmes? N'est-elle pas au contraire - et c'est probablement la définition même de la

16. Albin Michel 1963 et 1976; Paris, Librairie générale française (Le Livre de poche), 1984, p. 196-204. 
poésie - ce qui rend le langage possible?» (p. 204). Ainsi, la poésie est «ce qui rend le langage possible». La poésie a donc en commun avec le son de rendre le langage possible: le son est devenu poésie, et à la poésie il revient de prendre en charge l'ouverture au sein du sensible de la transcendance. L'essence du langage est donc poétique et la poésie continue d'assumer la sonorité du son. (b) Mais pour que la poésie relève de la transcendance du son, la déduction de la signification doit s'y faire immédiate, ou plutôt la signification procéder de la sonorité elle-même. C'est ce qu'indique Levinas au sujet du «Sonnet de voyelles» de Rimbaud, dans Humanisme de l'autre homme: Levinas évoque une signification qui serait produite par le son, où le son lui-même signifierait: "Des contenus sonores "dépourvus de sens" comme les voyelles, ont une "naissance latente" dans des significations - c'est là l'enseignement philosophique du célèbre sonnet de Rimbaud $»^{17}$. Les sons ne sont pas la base d'une signification, ils sont immédiatement et en eux-mêmes signifiants: dans la poésie, c'est le son lui-même qui prolifère en signification. (c) Mais la figure de Celan offre de cette relève poétique de la transcendance du son le développement le plus abouti: le poème pour Celan "se situe précisément à ce niveau pré-syntaxique et pré-logique (comme cela est, certes, de rigueur aujourd'hui), mais aussi pré-dévoilant»; c'est «le premier des langages», "plus ancien que celui de la vérité de l'être ${ }^{18}$. Commentons: d'une part, le poème, pour Celan, est pré-discursif, présyntaxique. Pourquoi? Parce que le poème donne naissance au langage, il est le lieu de genèse de la langue: nous rejoignons les considérations sur Claudel. Mais d'autre part, surtout, le poème n'est pas le lieu de la vérité de l'être et se situe avant le dévoilement. La portée polémique anti-heideggérienne de ces lignes est claire, puisque chez Heidegger, le poème dit l'être parce qu'au langage d'abord poétique revient la fonction de dévoiler l'être; or le poème comme son est pour Levinas le contraire du dévoilement et de la lumière, sortie de la phénoménalité à même la phénoménalité; nous sommes avant et hors l'être, avant le dévoilement, dans le premier des langages. C'est la genèse même de l'être à quoi nous assistons. Ainsi, en s'opposant à la lumière du dévoilement, le poème ouvre-t-il sur la langue même.

De cette façon revient-il au poème de ressusciter après 1948 l'origine de la parole telle que le son la déployait dans les écrits de 1948-1949. Cette relève poétique de la transcendance du son est, insistons-y, éminemment paradoxale, car le mot, tel qu'analysé jusqu'ici dans l'art, s'opposait au son par une double défaillance qui semblait doublement compromettre

17. Humanisme de l’autre homme [1972] Paris, Librairie générale française (Le Livre de poche - Biblio-Essais), 1987, p. 19-20.

18. Noms propres, Montpellier, Fata Morgana, 1976, p. 60. 
une telle relève. Dans l'œuvre d'art en général, Levinas aura montré que règne le silence neutre du il y $a$, de sorte que la poésie aurait dû, comme art, être associée à la neutralité de l'il y $a$, et non à la transcendance du son; d'autre part, dans le texte sur les Biffures de Leiris, Levinas se proposait de dépasser ou contredire le projet de Leiris, en opposant au mot-biffure le mot-son: en ce sens, derrière l'hommage se dissimulait une critique. La biffure de Leiris en restait à l'immanence, à la vision, et Leiris se plaçait incontestablement du côté artistique, occidental, immanentiste, du mot silencieux. Bref, un double discrédit sur l'art, à la fois général et régional, semblait disqualifier la poésie et lui interdire de prendre le relais du son. Mais ces deux objections portent court, ou plus précisément font voir très précisément l'enjeu: d'une part le rappel de disqualification de l'œuvre d'art gagne ici en nuance et en subtilité, puisque la mise en lumière de la fonction de transcendance de la poésie ne peut qu'inviter à nuancer la disqualification de l'art généralement attribuée à Levinas. D'autre part, si Leiris en restait au mot-biffure, Claudel, Rimbaud, Celan ouvraient le mot en sa transcendance. On pourrait ici schématiser en disant que Leiris occupe la face phénomène du son, et que Claudel, Rimbaud et Celan en occupent la face outre-phénomène. Tous les artistes ne se valent pas et la poésie fait chez Levinas l'objet d'une appréciation plus subtile qu'un simple discrédit massif, précoce et à tout prendre arbitraire.

Mais ces textes ne nous offrent pas seulement un appui pour soutenir la relève poétique de la phénoménologie du son, ils nous offrent aussi, au moins pour deux d'entre eux, une direction. Car reprenons-les: (a) dans le texte sur Claudel, Levinas évoquait l'" obligation de parler. Et si la politique, montant de partout, fausse les intentions originelles du discours, obligation de crier» (p. 204): la poésie, condition de possibilité du langage, satisfaisait ainsi aux intentions originelles du discours - c'est-à-dire à l'éthique. En repensant la poésie à partir de Claudel, Levinas fait de la poésie non seulement l'origine du langage, mais la gardienne des intentions originellement éthiques du discours. (b) S'agissant de Celan, pourquoi le poème s'ouvrait-il à la dimension pré-réflexive de la langue ? Précisément parce que le poème est lieu de l'éthique: «Langage de la proximité pour la proximité, plus ancien que celui de la vérité de l'être. Le premier des langages, réponse précédant la question, responsabilité pour le prochain, rendant possible, par son pour l'autre, toute la merveille du donner». Autrement dit, Celan se situe à un point où l'esthétique et l'éthique ne se distinguent pas encore: à l'origine des langues, donc à l'éthique comme philosophie première. Cette double analyse indique donc une ultime piste: le son, relayé par la poésie à laquelle il revient toujours de performer l'origine et l'essence du langage, se trouve pris en charge par la situation éthique elle-même, et en elle par le visage. 


\section{La relève éthique: le visage comme son}

Formulons nettement l'hypothèse: si le son disparaît après 1948, c'est pour laisser au visage sa place et sa fonction. En 1948, il revenait au son de trouer le monde, d'ouvrir sur la transcendance et de se muer en parole. En 1961, une telle fonction revient au visage. Pour valider cette hypothèse, il faut 1 / établir l'homologie entre le son et le visage et 2 / qu'une déduction du mot soit possible à partir du visage comme il l'était à partir du son (l'enseignement).

1/ Que le langage endosse les déterminations du son, c'est chose patente dans Totalité et Infini, et dès le début même de la section sur "Visage et extériorité», où Levinas propose de "distinguer regard et langage, c'est-àdire regard et accueil du visage» (p. 163): l'opposition regard / son de 1948 devient opposition regard/langage, ou plus exactement regard/visage. Suivons les points de comparaisons:

(a) De la même façon (et pour les mêmes raisons) qu'en 1948 le son en sa sonorité contredisait la vision, le visage s'établit en 1961 encore sur les ruines de la vision, compromise par son lien avec le toucher et l'espace, comme l'indiquent les pages $164-165^{19}:$ «La vision qui saisit à l'horizon ne rencontre pas un être à partir de l'au-delà de tout être» (p. 166). La vision, sens de la phénoménalité mondaine, n'ouvre que sur l'être heideggérien compris comme il y $a$, et ne permet pas de sortir de la transcendance ${ }^{20}$. Le visage s'imposera donc contre la vision, à la façon dont le son trouait le champ de vision en 1948.

19. Reprenons la critique de la vision dans Totalité et Infini : 1) «La lumière» de la vision «vide l'espace» (p. 163) et fait apparaître le il y a, fait apparaître les objets dans l'espace, c'est-à-dire l'ouvert comme condition d'apparition de l'objet, être saturé c'est-à-dire néant (p. 164); 2) cet espace est le lieu de la prise: «La vision s'ouvre sur une perspective, sur un horizon et décrit une distance infranchissable, invite la main au mouvement et au contact qui les assure» (p. 165). Comme il dit: «La vision se mue en prise». 3) Dès lors, «La vision n'est pas une transcendance. [...] Elle n'ouvre rien qui, par-delà le Même, serait absolument autre, c'est-à-dire en soi » : la vision n'ouvre qu'à la phénoménalité, c'est-à-dire la sphère du Même et «L'espace au lieu de transporter au-delà assure simplement la condition de la signification latérale des choses dans le Même» (p. 166).

20. C'est d'ailleurs ici que se décide à notre avis le sort de la différence ontologique dans Totalité et Infini: au sujet de la vision qui ouvre l'espace vide où apparaissent les choses, c'est-à-dire le néant, Levinas rappelle: «Dans la lumière de la généralité qui n’existe pas, s'établit la relation avec l'individuel. Chez Heidegger, une ouverture sur l'être qui n'est pas un être - qui n'est pas un "quelque chose" - est nécessaire pour que, d'une façon générale, un quelque chose se manifeste. [...] Ainsi apparaissent les articulations de la vision où le rapport du sujet avec l'objet se subordonne au rapport de l'objet avec le vide de l'ouverture qui, lui, n'est pas un objet. L'intelligence de l'étant consiste à aller au-delà de l'étant dans l'ouvert précisément. Comprendre l'être particulier c'est le saisir à partir d'un lieu éclairé qu'il ne remplit pas» (p. 164). Il s'agit là d'une référence à peine masquée à la différence ontologique: 1) l'ouvert, le vide, l'espace, conditionne l'objet (l'étant), au titre d'être. 2) ouvert par la vision, l'être se donne d'abord comme vide et néant, puisque la lumière «fait surgir précisément l'espace comme un vide». On reconnaît l'identification heideggérienne entre Être et Néant, posée dès Qu'est-ce que la métaphysique? (1929). Dès lors 3) l'identification 
(b) Le son était phénomène et non-phénomène, ce qui, depuis la phénoménalité, excède cette phénoménalité vers l'autre-du-monde. Or le visage assume précisément cette fonction, puisqu'il est le non-phénomène apparaissant à même la phénoménalité: «Encore pouvoir, car le visage s'exprime dans le sensible; mais déjà impuissance, parce que le visage déchire le sensible» (p. 172). Comme le son, le visage «n'est pas du monde» (p. 172), vient «d'au-delà du monde» (p. 190). Son ou visage, si transcendance il y a, elle est toujours à la fois d'une certaine manière phénoménalisée et non phénoménale, ou plutôt phénoménalisée à la manière d'un non-phénomène, puisqu'aussi bien faut-il que ce qui outrepasse les phénomènes encore s'y inscrive. Mais cette non-phénoménalité n’est pas en défaut sur le phénomène, mais bien en excès - excès constitutif de la transcendance. En ce sens, thématiser la sortie de la phénoménalité par le visage et faire de cette sortie un problème pour la phénoménologie a peut-être un sens pour l'examen de la phénoménologie, de ses méthodes et de ses limites, mais n'a pas de valeur critique quant à l'œuvre et au projet philosophique de Levinas: si le visage s'échappe (dans des conditions qu'il faudrait sans doute encore préciser) de la phénoménologie, ce n'est pas par une insuffisance de la phénoménologie, mais parce qu'il revient à la transcendance de sortir en quelque manière des phénomènes.

(c) Troisième critère: en 1948, Levinas soutenait que dans le phénomène du monde, la forme clarifiait le contenu et lui prête un sens, de sorte que ce contenu lui-même devenait un fait de vision. Il appartenait au son en revanche, comme sa prérogative propre, «que de déborder sa forme et de ne pas nous donner un autre monde qui en tant que lumière serait englobé dans celui où nous sommes ${ }^{21}$. Dans le son, le contenant (sensible) ne contient pas le contenu (extra-sensible, extra-mondain), qui déborde vers l'autre-du-monde. Or Levinas ne dira pas autre chose s'agissant du visage. En effet, le visage lui-même n'admet pas de forme, car «sous la forme, les choses se cachent ${ }^{22}$. Dès lors, "Si le transcendant tranche sur la sensibilité, s'il est ouverture par excellence, si sa vision est la vision même de l'ouverture elle-même de l'être - elle tranche sur la vision des formes et ne peut se dire en termes de contemplation» (p. 172): «Le visage, encore chose parmi les

heideggérienne de l'être et du néant et de la brutalité de l'il y a (p. 165), auquel emprunte toutefois l'élémental de la jouissance (selon des modalités particulières), disqualifie l'être de l'étant comme provenance de l'étant. Levinas répondra à cela par: "Au dévoilement de l'être en général, comme base de la connaissance et comme sens de l'être, préexiste la relation avec l'étant qui s'exprime; au plan de l'ontologie, le plan éthique» (p. 175). La différence ontologique est ainsi contestée par le primat de l'étant dans l'éthique.

21. "Parole et Silence», p. 90.

22. Totalité et Infini, p. 166. 
choses, perce la forme qui cependant le délimite» (ibid.). Le visage perce la forme qui le délimite, parce qu'il l'excède et l'outrepasse.

(d) Comme le son, le visage devient (non-)phénomène de l'événement autre. Le visage est «l'événement de l'altérité». Il faudrait en conclure que, comme le son dont l'essence est verbe, le visage est verbe. Levinas va-t-il jusque-là ? Oui, sans conteste: «Se manifester comme visage, c'est s'imposer par-delà la forme, manifestée et purement phénoménale, se présenter d'une façon, irréductible à la manifestation» (p. 174). Il y a donc bien, à travers le visage, l'événement éthique ou événement de la socialité - et même plutôt éthique et socialité comme événements donnés par le visage: «l'accueil de l'être qui apparaît dans le visage, l'événement éthique de la socialité, commande déjà le discours intérieur» (p. 182; c'est l'événement éthique); «la présentation du visage me met en rapport avec l'être. L'exister de cet être - irréductible à la phénoménalité [...] - s'effectue dans l'inajournable urgence avec laquelle il exige une réponse» (p. 187). Bref, par le visage perce autrui, "Autrui, inséparable de l'événement même de la transcendance» (p. 210).

2/ Soit la seconde condition: si le son devient visage, il doit s'ensuivre une déduction possible du langage à partir du visage, une conversion du visage en langage, tout comme le mot s'ensuivait de l'essence du son. Levinas aura précisément décrit cette conversion sous l'aspect d'une genèse de la signification sur laquelle nous ne nous attarderons pas, s'il est vrai que le visage est condition "présupposée» par langage («accueil du visage que le langage présuppose», p. 163), et que c'est le visage qui «ouvre» le discours (le «discours qu'ouvre l'épiphanie comme visage», p. 175). En tant qu'ouvert originellement par le visage, le langage n'est pas un instrument pour communiquer une pensée préalablement secrète ou pour manipuler le monde, mais expression: « ... bien que le langage puisse s'intégrer à un système d'actes et servir d'instrument. Mais le langage n'est possible que lorsque la parole renonce précisément à cette fonction d'acte et lorsqu'elle retourne à son essence d'expression" (p. 176). À l'expression revient donc l'essence du langage, expression du visage. Nous avons vu que l'essence du langage était poétique, nous voyons à présent qu'elle est fondamentalement expression - c'est-à-dire déduite du visage, tout comme le mot était déduit du son. Une telle thèse a naturellement une portée critique extrêmement puissante, puisqu'elle contredit la genèse heideggérienne de la signification par la mondanéité du monde ${ }^{23}$, lui substituant une genèse par l'expression à partir du visage ${ }^{24}$.

23. Voir Sein und Zeit, $\$ 18$.

24. Cette déduction du langage à partir du visage au moyen de l'expression s'atteste encore de l'essence symbolique de la signification. Il revient à la page 182 de Totalité et Infini de donner 
Ce point est donc acquis: le visage endosse dans Totalité et Infini les fonctions et les prérogatives du son en 1948. Cette thèse est-elle tout à fait satisfaisante? Oui, si l'on admet la communauté de description entre l'un et l'autre; mais cette solution ne doit pourtant pas être poussée trop loin, car elle manque le formidable "resserrement» des catégories descriptives dans Totalité et Infini - double resserrement si l'on peut dire: (a) resserrement entre le visage, l'expression et le langage. Alors que la conférence de 1948 déduisait le mot à partir du son, lui-même analysé en son essence (la sonorité), le visage est lui-même la parole qui est expression : "Cet infini, plus fort que le meurtre, nous résiste déjà dans son visage, est son visage, est l'expression originelle, est le premier mot: "tu ne commettras point de meurtre"» (p. 173). Le visage est lui-même expression, comme il est lui-même parole (p. 176: «comme visage, c'est-à-dire comme parole»). C'est pourquoi le langage lui-même fait événement au lieu d'être symbole de l'événement autre: «Le langage... constitue un événement irréductible à la conscience où tout survient de l'intérieur» (p. 179). Autrui est alors reconduit essentiellement à sa signification, il est sa signification: "La signification c'est l'infini, c'est-à-dire Autrui...» (p. 182). On le voit: ce qui se déployait en plusieurs étapes en 1948 se concentre en 1961 en un événement unique, le visageexpression-langage-infini. La catégorie de déduction laisse la place à une équivalence stricte. Ce premier resserrement n'est toutefois problématique que dans une philosophie de type dialectique, au sein de laquelle les moments sont à envisager comme à la fois essentiellement distincts et ouvrant l'un sur l'autre: dans un tel schéma, la distinction des moments descriptifs est la contrepartie paradoxale de la dépendance des instances déduites et de leur commune dépendance au temps jusqu'à l'Universel Concret. Mais en bonne phénoménologie, la description lévinassienne n’isole des moments que descriptivement, sans supposer leur distinction essentielle. Les moments de la description du visage n'ôtent rien à l'unité du phénomène lui-même. 2) Deuxième resserrement, plus décisif peut-être et plus subtil: l'absence de médiation entre moi et le visage. En 1948, le son ouvrait sur le visage, alors qu'il est le visage en 1961. Relisons «Parole et Silence», p. 93: "le langage [comme son], c'est la possibilité pour un être d'apparaître du dehors, pour

du symbole la définition la plus nette, clarifiant (et même contredisant à certains égards) les développements encore inchoatifs de "Parole et Silence» : «En un sens, la signification est à la perception ce que le symbole est à l'objet symbolisé. Le symbole désigne l'inadéquation du donné de la conscience à l'être qu'il symbolise, une conscience besogneuse et affamée de l'être qui lui manque, de l'être qui s'annonce dans la précision même avec laquelle est vécue son absence, une puissance qui pressent l'acte. La signification lui ressemble, comme débordement de l'intention qui vise, par l'être visé». Or en étant symbole, le langage ouvert par le visage récupère ce qu'il lui appartenait comme son en 1948. 
une raison d'être toi, de se présenter comme visage, tentation et impossibilité du meurtre». Ainsi le son était-il une voie d'accès au visage, comme trouée de l'immanence vers autre chose, porte ouverte, mais ouverte sur autre chose qu'elle-même: le son assumait une fonction médiatrice. De cette fonction médiatrice s'ensuivait une curieuse, paradoxale mais implacable conséquence: par une nécessité phénoménologique, le visage comme tel, bien qu'alors évoqué, ne pouvait faire l'objet d'une description, sa mention ne pouvait pas se développer en une description plus riche, puisque les déterminations à venir du visage n'étaient encore que celles du son. D'autant plus au son, d'autant moins au visage: c'est-à-dire, en 1948, rien au visage, nous regardions le doigt qui nous montre la lune et non encore la lune, puisque seul le doigt confisque à la lune sa lumière. Le visage évoqué dans le texte de 1948 n'est pas encore - et ne peut pas être - le visage de 1961. Pourquoi Levinas n'a-t-il pas conservé au son sa fonction médiatrice? Pour une raison que nous avons rencontrée, à savoir parce que dans Totalité et Infini la sensation est jouissance, intériorité, et que la transcendance doit en passer par autre chose que la sensation, il ne s'agit plus de favoriser le son comme l'autre de la lumière, l'audition comme l'autre de la vue, mais de promouvoir le visage comme autre de toute sensation, fût-ce de l'audition elle-même.

\section{Conclusions}

Plusieurs conclusions s'imposent.

1/ Si l'on suit la progression de l'élaboration de Levinas, il apparaît que le visage est pensé à partir $d u$ son, c'est-à-dire à partir du langage. De 1948 à 1961, Levinas a transféré les caractères du son au visage, pensant le visage à partir du son, et reléguant dans Totalité et Infini le son à l'immanence de la sensorialité. Ainsi, nous pourrions dire que le premier visage du visage, c'est le son; ou même: que le son fut tout simplement le premier visage, puisqu'en ouvrant sur le visage, comme le proposait le texte de 1948, il n'ouvrait sur rien qui pût être décrit. On comprend dès lors que, dans Totalité et Infini, sans la médiation de la sensation (fût-ce du son), le visage doive s'imposer de lui-même. Suivre l'élaboration du concept de visage à travers les inédits serait une tâche à part entière et à laquelle en un sens la présente communication voudrait collaborer, mais un rapide examen des Carnets de captivité du volume 1 indique que le visage est construit plus tard que le son, et à partir de lui. Le son apparaît dès 1944, à partir d'une méditation sur l'opposition du silence et le verbe ${ }^{25}$, sur la sonorité

25. On ne pourra à titre indicatif que renvoyer sans commentaire à l'extrait suivant: «Le silence et le verbe. Esprit européen: le verbe plus fort que le silence. Mais qu'est-ce que le verbe 
du son ${ }^{26}$ puis sur la verbalité de l'être, donnée dans le son ${ }^{27}$. Le visage apparaît plus tard et ne connaît son plein déploiement que dans les Notes philosophiques diverses ${ }^{28}$, au moment où le son lui-même est abandonné au profit des analyses sur l'enseignement. Il en va de même pour les œuvres publiées: le visage tel qu'il sera décrit dans Totalité et Infini est relativement tardif dans les œuvres publiées, puisqu'il n'apparaît pas dans De l'évasion (1935) ni dans De l'existence à l'existant (1947) ${ }^{29}$; c'est dans Le Temps et l'Autre (1946-1947) que le concept semble s'esquisser, encore noyé dans une paraphrase qui se cherche ${ }^{30}$, attendant son déploiement définitif à partir de Liberté et commandement (1953). Il y aurait donc substitution d'une topique du visage à une topique du son autour du changement de décennie.

2/ La fortune du son est donc dépendante, dans le déploiement de la pensée lévinassienne, du sort dévolu à la sensibilité. En 1948, la sensibilité est bien déploiement de l'immanence du sujet, mais le son est le seul phénomène perceptible par elle qui déroge à son régime commun de fonctionnement: toute sensorialité relève de la vision en ce que la vision orchestre le perçu comme relevant de l'immanence, à l'exception du son, qui, par un de ses

originellement? Pensée? Non: expression - la manière dont l'intérieur devient réalité. Accomplissement. Le silence n'est pas réalité - Mais intervalle» (p. 133).

26. La page 145 des Euvres 2 contient un passage où la méditation sur le son est déjà acquise: l'essentiel est déjà acquis.

27. Voir Carnets de captivité, p. 166: «L'Être n'est pas seulement un verbe - c'est le Verbe. [...] Le verbe c'est le son - Le son en tant que retentissement - vibration - ce qu'il y a d'action dans la vibration - se maintenir - une certaine amplitude - D'où différence entre voir - comprendre et entendre - (il faut évidemment ponctuer «voir - comprendre et entendre », plutôt que "voir - comprendre et entendre», comme le proposent les éditeurs). Et page suivante: «Le son - pour réapparaître doit être reproduit. Le rouge est le même rouge - mais le do est toujours nouveau. Certes le rouge aussi se reproduit à nouveau avec chaque aube - mais il ne recommence pas comme le son - c'est la lumière qui revient tandis que pour le son $<c^{\prime}$ est $>$ tout son être qui se refait. / Être = verbe. Il y a une question préliminaire: les mots - sont-ils des noms, c'.-à-d. même le verbe un nom d'action, adjectif = nom de qualité, etc. ou au contraire les noms eux-mêmes sont-ils des verbes polarisés? L'essence du mot n'est ce pas le verbe? Dès lors lien intime entre mot et le verbe être. / Le verbe d'être - dilatation, contraction de l'être./ Analyse de la durée en tant qu'être [...] mais eros ou verbe d'être - ou son".

28. Voir Euvres 1, p. 268: «L'expérience du visage - est la relation dissymétrique »; la page 280 contient une nouvelle élaboration du visage; cf. aussi, par exemple, p. 369 , p. 371. Il semble que ce soit dans les années des Notes philosophiques diverses qu'apparaît le concept rigoureux de visage, soit dans les années 1950 - donc après les méditations sur le son.

29. On y trouve bien deux occurrences de «visage», mais qui n'ont rien à voir avec l'invisible apparaître d'autrui.

30. "Cette situation où l'événement arrive à un sujet qui ne l'assume pas, qui ne peut rien pouvoir à son égard, mais où cependant il est en face de lui d'une certaine façon, c'est la relation à autrui, le face-à-face avec autrui, la rencontre d'un visage qui, à la fois, donne et dérobe autrui» (Le Temps et l'Autre, p. 67). 
aspects (la sonorité), y échappe et indique la transcendance. En 1961, la reconduction définitive et universelle de la sensibilité au moment du Moi (dans la jouissance) met un terme à l'exception du son développée en 1948: le son se laisse décrire comme tout sensible, relevant de la sphère du moi; dès lors, la trouée de la transcendance ne se fait plus par une quelconque exception au régime commun de la sensorialité (le son), puisqu'une telle exception n'existe pas, mais en passe par un phénomène qui ne relève d'aucune sensibilité (le visage nouménal). Enfin, la réintégration du son comme marqueur de transcendance ne pourra se faire, dans Autrement qu'être ou au-delà de l'essence, qu'au moyen d'une réévaluation globale et générale du rôle de la sensibilité et d'une réhabilitation de la sensorialité, puisque, en 1974, elle sera décrite comme une forme de passivité déjà indice de la transcendance.

3/ Si l'essence du langage demeure déposée dans la poésie et dans l'éthique, force est de relativiser le discrédit dont est frappée l'esthétique chez Levinas. La double relève, à la fois poétique et éthique, de l'analyse du son rend possible une esthétique de l'éthique et une éthique de l'esthétique, loin des lieux communs sur l'opposition de Levinas à l'art. Cette reconsidération de la place des arts doit se faire d'abord par la poésie, et, peut-être, par la musique - et relègue à nos préjugés spontanément picturalistes la thèse d'une opposition de Levinas à l'art. Les sons n'échappent sans doute pas à leur "qualification", c'est-à-dire à leur pétrification musicale ou linguistique, mais la musique comme la poésie peuvent tenter d'échapper elles-mêmes à la dictature de l'immanence.

4/ Penser le visage à partir du son d'une part, resserrer dans le phénomène du visage le langage et l'expression d'autre part, voilà qui permet de penser le visage comme son - c'est-à-dire comme appel - et «conscience comprise comme audition ${ }^{31}$. Dans la mutation du son en visage, il y a plus qu'un changement de topique affectant le déploiement d'une œuvre (même si c'est aussi un changement de topique): un véritable tour de force consistant à promouvoir le concept paradoxal d'un visage qui s'entend. Ainsi, le visage est invisible; en rigueur, nous devrions ajouter qu'il ne s'entend pas davantage - n'était qu'il fut d'abord son, et qu'il fut audible avant d'être visage. L'illustration la plus exacte de ce visage qui s'entend est le cri entendu par Jean-Baptiste Clamance dans la Chute de Camus, appel de la transcendance au sein de la nuit obscure du neutre ${ }^{32}$. Ainsi, la phénoménologie déserte le

31. Hors Sujet, p. 201.

32. Il y aurait une comparaison à mener entre la situation éthique chez Levinas et l'événement initiateur de la "chute» de Clamence (La Chute, in Théâtre, récits, nouvelles, Paris, Gallimard (Pléiade), 1985, p. 1511): depuis la situation de l’intériorité jouissante («J'étais 
visible et s'attache à penser le (contre-)phénomène comme ce qui s'entend plutôt que comme ce qui se voit ${ }^{33}$. La subjectivité de l'autre du Dasein (le Juif) n'est pas celle de l'homme qui voit, mais celle de l'homme qui entend ${ }^{34}$, et c'est pourquoi elle s'épanouit dans le modèle du prophète biblique. Encore faut-il pourtant ici faire une précision, suggérer une remarque et indiquer un paradoxe. La précision: si le visage s'impose de lui-même, endossant les caractéristiques du son tout en en révoquant la médiation, alors l'appel en quoi il consiste ne s'entend que par métaphore; c'est le son qu'aucun instrument ne saurait produire, qu'aucun sonomètre ne saurait mesurer. La remarque: en déterminant l'événement d'autrui comme son et la subjectivité comme audition, Levinas déplace le concept d'appel depuis la subjectivité immanente et silencieuse où l'avait logé Heidegger vers la transcendance sonore de l'Autre ${ }^{35}$. Le paradoxe: si une phénoménologie lévinassienne ou post-lévinassienne est possible, elle ne peut l'être qu'en explorant le phénomène non phénoménal dans la direction de sa sonorité: la sortie de la subjectivité transcendantale (dont l'analyse du Dasein n'offre peut-être que la dernière figure) ne peut en passer que par la contestation du primat de la vision et la promotion de l'audition comme concept phénoménologiquement supérieur et peut-être décisif. Le révélé n'est pas le visible, mais l'entendu. Le visible ou le révélé: la disjonction est exclusive, il faut choisir ${ }^{36}$.

5/ La cinquième et dernière conclusion rejoindra la précédente. La pensée du visage comme invisible et comme son interdit toute pensée de l'Incarnation et nourrit la spéculation anti-chrétienne des années 1940-1950. Quelle qu'ait été la position de Levinas sur le christianisme (et elle semble

heureux de cette marche, un peu engourdi, le corps calmé, irrigué par un sang doux»), jusqu'à l'anonymat de la femme suicidaire, à peine décrite, réduite à sa «nuque, fraîche et mouillée», puis à son "cri, plusieurs fois répété» - on songe évidemment à la nuque comme visage, dans Éthique et infini. Quant au «silence qui suivit, dans la nuit soudain figée», il se laisse lire comme le triomphe de l'être anonyme sur l'éthique. Mais, à notre connaissance, la confrontation entre La chute et l'œuvre de Levinas, sans doute suggestive et féconde, attend encore son élaboration systématique.

33. Voir Totalité et Infini: «le visage en appelle à moi» (p. 168); «Se manifester en assistant à sa manifestation revient à invoquer l'interlocuteur» (p. 174).

34. Voir Totalité et Infini: «Le visage où autrui se tourne vers moi, ne se résorbe pas dans la représentation du visage. Entendre sa misère qui crie justice ne consiste pas à se représenter une image, mais à se poser comme responsable» (p. 190).

35. Voir Être et Temps, $\$ 57$, p. 275: «Dans la conscience, le Dasein s'appelle lui-même»; et cet appel lui-même est silencieux: «La conscience parle uniquement et constamment sur le mode du faire-silence» (Etre et Temps, $\$ 56$, p. 274). Ce silence, qui tire le Dasein du bavardage du $O n$, est reconduit chez Levinas à l'immanence de l'il $y a$.

36. Ainsi nous semble-t-il que la postérité lévinassienne doit, de ce point de vue, davantage être trouvée dans L'appel et la réponse (Paris, Minuit, 1992) ou Répondre: figures de la réponse et de la responsabilité (Paris, PUF, 2007), de Jean-Louis Chrétien, que dans La croisée du visible (Paris, PUF, 1991) et Le visible et le révélé (Paris, Cerf, 2005), de Jean-Luc Marion. 
avoir été subtile, évolutive), quelles que soient les perspectives théologiques ouvertes par son travail (elles sont grandes), il faut admettre que la pensée du son comme premier visage du visage interdit toute inscription de la phénoménologie lévinassienne dans un horizon chrétien. Le texte sur Leiris l'énonce clairement: «dans le rapport social, la présence réelle de l'autre importe; mais, surtout, [...] cette présence s'accomplit dans l'audition, tire son sens de ce rôle d'origine transcendante que joue la parole proférée. C'est en tant que le verbe se refuse à devenir chair qu'il assure une présence parmi nous » (p. 202). La parole est son, mais son qui se refuse à devenir chair, qui ne doit pas le devenir. Pourquoi ? Parce que par la chair, le son perdrait sa transcendance même: «Dans la parole - qui part d'un corps - se révèle une idée séparée de son incarnation. La parole est dans ce sens principe de l'abstraction. La parole est donc la vraie façon pour Dieu de se révéler. L'incarnation est aux antipodes de la révélation ${ }^{37}$. Ainsi s'établit encore une véritable opposition au christianisme: "pour le judaïsme \{Dieu\} ne s'incarne pas. Non pas parce qu'un tel événement serait choquant pour la raison, mais parce qu'il placerait la perception au-dessus $\{$ du verbe $\}$ et compromettrait l'intériorité ${ }^{38}$. Si le visage est pensé à partir du son et que le son demeure réfractaire à toute incarnation, alors la phénoménologie du visage a tout d'une opposition à l'Incarnation chrétienne. En ce sens, penser la Révélation revient à contredire le christianisme. Le visage est l'invisible devant rester invisible; une pensée de l'Incarnation ne serait alors possible qu'à la condition d'enfreindre l'interdit de visibilité, en restaurant, par-delà Levinas et avec Heidegger, la visibilité comme détermination phénoménologique première. Mais alors, écrire qu'à travers le visage, "la chair se fait verbe, la caresse - Dire ${ }^{39}$, sera-ce encore contredire le christianisme en renversant la formule de Jean I 14, ou au contraire est-ce réintégrer la chair et l'unir au Verbe? À moins que Levinas n'ait tenté de (donner à) penser et à voir l'invisibilité du visible.

Dan ArbiB

Fondation Thiers - CNRS

37. Euvres 1, p. 413-414.

38. Ibid., p. 255.

39. Autrement qu'être ou au-delà de l'essence, La Haye, Martinus Nijhoff, 1974, p. 125, et Humanisme de l'autre homme, p. 48. 